\section{La nécessité d'un programme d'amélioration de certaines essences forestières feuillues: Applications aux genres Juglans et Acer}

\author{
Par L. PARROT
}

Faculté de Foresterie et de Géodésie, Université Laval, Québec ODC 165.3

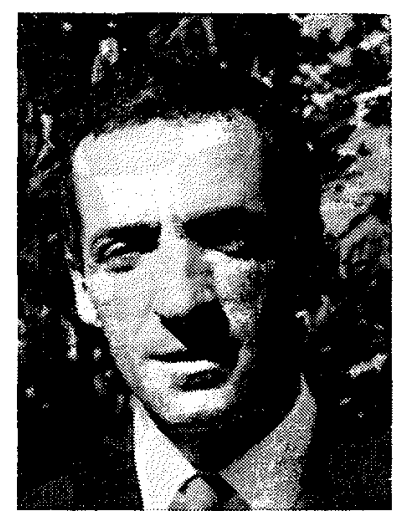

\section{Résumé}

Devant le nombre des travaux de recherche effectués en amélioration des arbres forestiers retenant les essences résineuses, il ne faut pas pour autant négliger les espèces feuillues de valeur qui sont exploitées en nos régions. La nécessité d'établir des programmes d'amélioration pour ces espèces forestières est indiscutable. De tels programmes devraient être, dans la mesure du possible, basés sur des études génécologiques.

Un premier exemple puisé chez le Juglans nigra permet de tirer des conclusions d'une adaptation génétique fort variable pour cette espèce forestière qui conduisent à des résultats d'ordre pratique.

Ces résultats sont obtenus en plus au moyen d'une étude à court terme de 17 provenances décrivant la variation de leur rusticité sous la latitude du lieu de culture.

Une autre espèce plus autochtone en nos régions que la précédente, soit Juglans cinerea, également fort recherchée en ébénisterie et en sculpture est actuellement étudiée au point de vue de sa variation infraspécifique. Les résultats qui en découleront permettront de sélectionner les meilleures régions d'utilisation des semences. Un tel programme d'amélioration pourrait éventuellement alimenter plus d'une petite industrie locale.

Un second exemple utilisant l'Acer saccharum, décrit les résultats d'une recherche quinquennale effectuée dans le but d'étudier la variation de la teneur en sucre de la sève. A cette fin, 3 érablières furent visitées à chaque printemps de 1965 à 1969 .

L'homogénéité d'une part des résultats obtenus dans 2 érablières conduit à une variation très faible comparativement ̀̀ celle existant dans le troisième peuplement. En effet, l'importance de la variation maintenue constante clurant ces années conduit à une sélection de neuf génotypes dont la teneur en sucre varie de $4.6 \%$ à $9.7 \%$ indiquant pour certains individus un gain génétique de l'ordre de $150 \%$.

La continuation de recherches en propagation végétative de l'Acer saccharum est nécessaire afin de construire avec plus de facilité les populations clonales requises à un tel programme d'amélioration.

\section{Abstract \\ The Need For A Tree-Breeding Program For Certain Hardwood-Forest Species: Applications In The Genera Juglans and Acer}

Although most of our tree-breeding programs are concerned with coniferous forest species, we must not neglect the valuable hardwoods exploited in our region. The need of these programs for hardwoods is undeniable and they should be based, as much as possible, on genecological studies.

One such study illustrates the wide variation in genetic adaptation of Juglans nigra and indicates its practical implications. Further, these results were obtained from a short-term study of 17 provenances for variations in frost-hardiness at a single location.

Intra-specific variation is being investigated in Juglans cinerea, a more common species in our: region than the one previously mentioned and one which is also in strong demand for furnituremaliing and wood sculpture. The resulting data will permit the selection of the best seed zones. Such a breeding program could eventually support far more than small, local industries.

Another project involving Acer saccharum describes the variation in sugar-content of sap in three maple stands sampled each spring from 1965 to 1969. Two stands vere very homogeneous compared to the third. The magnitude of the differences maintained throughout the study period led to the selection of nine genotypes whose 
sugar contents varied from $4.6 \%$ to $9.7 \%$, in dicating maximum genetic gains on the order of $150 \%$.

Continued research in vegetative propagation of Acer saccharum is needed for the production of clonal populations for breeding programs.

\section{1- Avant-propos}

L'optique des programmes d'amélioration varie d'un continent à l'autre, d'un pays à l'autre ou d'une grande région écologique à une autre. Certains organismes, de par le monde et par l'intermédiaire de leurs sylviculteurs et généticiens, réussissent à concrétiser leurs recherches sur un grand nombre d'espèces résineuses (Pinus, Picea, Larix). Les résultats obtenus depuis les deux dernières décennies montrent des gains génétiques et économiques indiscutables. D'autres mettent l'accent sur les essences feuillues à croissance rapide, tels les Populus et les Eucalyptus. Dans ce dernier cas, les problèmes d'adaptation au nouveau milieu d'utilisation sont d'une extrême importance lorsqu'on fonde un programme d'amélioration employant une espèce exotique. Au Canada, où la grande majorité des forêts est constituée de conifères, la plupart des travaux de génétique forestière s'orientent vers les résineux de sorte que très peu de travaux d'amélioration (recherche fondamentale ou recherche appliquée) s'effectuent dans le secteur des feuillus, à l'exception du genre Populus. Si d'un point de vue écologique l'aire des feuillus de grande valeur (Tilia, Fraxinus, Juglans, Quercus et Betula) est inférieure à celle des conifères, l'industrie des bois francs, proportions gardées, n'en est pas moins aussi importante. II ne faut pas pour autant négliger les domaines de l'ébénisterie et de la sculpture qui doivent souvent s'approvisionner en matière première à l'extérieur du pays.

Les différentes études mondiales prévoient une pénurie de matière ligneuse d'ici quelque 30 ans. Si l'accroissement de la demande est prévu suffisamment à l'avance, on peut prévoir l'établissement d'une forêt de la dimension voulue sur un lieu approprié. Ceci pose certaines difficultés, mais il ne faut pas nier les difficultés pour les abolir. II faut créer des conditions telles que l'on puisse affirmer avec une certaine assurance que ces problèmes ne se poseront pas. Que fait-on pour conserver et améliorer les essences feuillues sous notre latitude? Les efforts en ce sens pratiqués dans le sud de l'Ontario sont fort louables (10) même s'ils furent effectués sans motifs de recherche au début. Les résultats indiquent combien les connaissances de l'adaptation génétique, de la plasticité et variabilité d'une espèce vis-à-vis les différents lieux de culture sont indispensables à une réussite des programmes de boisement. Les connaissances actuelles de ces résultats permettraient d'atteindre à l'avenir de meilleures réussites des reboisements de feuillus. Cette proportion d'échecs et de réussites mentionnée par l'auteur, mise en parallèle avec les récentes applications de la génétique forestière, servira de clés à une meilleure rationalisation des essences feuillues à être intégrées dans de tels programmes.
Un bref retour au coeur des vieux pays européens caractérisés par une sylviculture plus que centenaire en certains cas, nous donne des exemples fort intéressants d'essais de vieux reboisements dont certains pratiqués au moyen de plants de Pinus laricio greffés (1), et de Quercus. D'autres exemples peuvent être cités concernant le Pinus radiata bouturé sur une grande échelle (6).

La question peut être soulevée: peut-on apporter une certaine amélioration aux arbres sans recherche ou, peut-on tirer des résultats valables de tels essais vieux de près de 100 ans et faits souvent par simple curiosité ou goût des arbres? De nos jours, il est reconnu que le "tree breeding" peut aider à améliorer la production forestière une fois la recherche effectuée pour une espèce donnée. Par contre, quelles sont parmi nos espèces forestières celles pour lesquelles des études et analyses génétiques sont complètes, voire même partiellement complétées? C'est un phénomène rare. Les Eucalyptus, espèce prolifique fort populaire en Afrique et en Asie, furent tout récemment introduits en Tunisie sur 30,000 hectares sans étude génécologique ou comparative préalable. Cette introduction se solda en un échec total.

\section{2- La génécologie}

Si l'on parle de reboisement ou de boisement, des études génécologiques sont indispensables comme recherche de base. Cette science, la génécologie (la génétique écologique appliquée au monde végétal) s'occupe de l'étude des provenances et traite surtout de la variabilité infra-spécifique, en l'occurrence, des populations d'arbres d'une même espèce étudiées en milieux naturels variés ou celles-ci se développent et vivent. Elle recherche en définitive les modalités d'adaptation de populations évoluant en des régions différentes. D'un point de vue pratique, ces études génécologiques terminées, les résultats qui en découlent permettent de sélectionner des peuplements constitués en "banque de semences" produisant des arbres bien adaptés au lieu de culture et caractérisés par une productivité forte et qualitative, un des principaux critères recherchés dans tout programme de reboisement ou de boisement.

\section{3- Une étude du genre Juglans}

Dans la province de Québec, un exemple frappant d'une vieille plantation effectuée en 1882, près de Lotbinière, nous donne aujourd'hui un résultat concret pouvant servir de base à un programme d'amélioration bien qu'il soit évident que l'auteur de ce reboisement ne songeait nullement à faire une étude quelconque de provenances de noyer noir.

Cette espèce ligneuse, pratiquement exotique au Canada, à l'exception d'une aire très restreinte, limitée au sud de l'Ontario, se montre exigeante et préfère les sols riches et profonds non exposés aux vents prédominants.

Le tableau I donne la liste descriptive des provenances introduites en 1882 .

Tableau I - Liste des provenances introduites en 1882. 
Tableau I - Liste des provenances introduites en 1882 .

\begin{tabular}{|c|c|c|c|c|c|}
\hline Pays & Localité & Province & $\begin{array}{l}\text { Alti- } \\
\text { tude }\end{array}$ & $\begin{array}{l}\text { Lati- } \\
\text { tude }\end{array}$ & $\begin{array}{l}\text { Longi- } \\
\text { tude }\end{array}$ \\
\hline Canada & Wardsville & Ontario & $700^{\prime}$ & $42^{\circ} 39^{\prime}$ & $81^{\circ} 45^{\prime}$ \\
\hline Canada & Simcoe & Ontario & $650^{\prime}$ & $42^{\circ} 50^{\prime}$ & $80^{\circ} 20^{\prime}$ \\
\hline Canada & $\begin{array}{l}\text { Montréal } \\
\text { (introduit) }\end{array}$ & Québec & $140^{\prime}$ & $45^{\circ} 24^{\prime}$ & $72^{\circ} 36^{\prime}$ \\
\hline E.U. & - & Wisconsin & - & $42^{\circ} 20^{\prime}(?)$ & $89^{\circ} 20^{\prime}(?)$ \\
\hline
\end{tabular}

Seule la provenance du Wisconsin n'a pas d'origine connue.

\section{a) Résultats actuels}

Les 265 tiges qui ont résisté jusqu'à nos jours se répartissent en six groupes composés d'un nombre variable d'individus. Ainsi plantés sous cette latitude nord de $46^{\circ} 40^{\prime}$, soit quelque 210 milles plus au nord que l'extrême limite de l'aire fixée au $43^{\circ}$, et sur deux catégories de sols, bon nombre de semis ne purent résister à plus d'un hiver et moururent. (5) L'influence de la sélection naturelle fut drastique en certains cas et son action s'est perpétuée jusqu'à nos jours, modelant de façon irrégulière cet ensemble de populations.

La résistance des tiges au froid est de fait très variée et permet un classement de la rusticité des arbres allant de sujets parfaitement sains et adaptés, d'une hauteur variant de 70 à 95 pieds, et produisant un volume ligneux analogue à celui des états américains, où le noyer noir atteint alors une forte productivité, à des individus génétiquement mal adaptés et caractérisés par un tronc plus ou moins avarié par des gélivures. De tels individus atteignent une hauteur du tiers inférieur à celle des tiges ayant cru normalement. (9)

\section{b) Une étude de provenances}

La presque totalité de la plantation fut établie sur des sols et sites répondant aux exigences du noyer noir et les meilleurs phénotypes ont été sélectionnés pour établir un programme d'amélioration. Toutefois, le nombre de provenances de cette vieille plantation beaucoup trop restreint et réduit par la sélection naturelle ne pouvait à lui seul représenter une population suffisante de l'aire totale permettant de tirer des conclusions acceptables pour un tel prógramme. Ainsi, une étude à court terme a été mise sur pied groupant au total 17 provenances examinées durant les années -1967 et 1968.

Le tableau II donne la liste des provenances ainsi que les dommages dus au froid, caractérisés par un noircissement d'une partie ou de la tige entière qu'accompagne un desséchement des bourgeons. Ces provenances se groupent dans l'ensemble de l'aire naturelle exception faite de celle de la Colombie Britannique, à climat continental et introduite en cette région il y a plus de 50 ans. (4)

Les résultats du tableau II montrent qu'il existe une relation nette entre la latitude du lieu d'origine de la provenance et la susceptibilité au froid. Cette
Tableau II - Semis de 2 ans endommagés par le froid durant les hivers 1967 et 1968. (Inventaire du 20 juin 1968)

\begin{tabular}{|c|c|c|c|c|c|}
\hline $\begin{array}{l}N^{\circ} \text { pro- } \\
\text { venance }\end{array}$ & Origine & $\begin{array}{l}\text { Lati- } \\
\text { tude }\end{array}$ & $\begin{array}{l}\text { Longi- } \\
\text { tude }\end{array}$ & $\begin{array}{l}\text { Total } \\
\text { des } \\
\text { semis }\end{array}$ & $\begin{array}{l}\text { \% semis } \\
\text { endom- } \\
\text { magés }\end{array}$ \\
\hline 40 & Madison Co. Tenn. & $35^{\circ} 41^{\prime}$ & $88^{\circ} 43^{\prime}$ & 148 & 87 \\
\hline 25 & Licking, Miss. & $37^{\circ} 38^{\prime}$ & $92^{\circ} 53^{\prime}$ & 328 & 58 \\
\hline 43 & Dawson Spgs, Dent & $37^{\circ} 32^{i}$ & $85^{\circ} 18^{\prime}$ & 130 & 61 \\
\hline 48 & Carbondale, Ill. & $37^{\circ} 50^{\prime}$ & $89^{\circ} 30^{\prime}$ & 60 & 64 \\
\hline 28 & Augusta Co., Va. & $38^{\circ} 15^{\prime}$ & $78^{\circ} 45^{\prime}$ & 74 & 49 \\
\hline 47 & Manhattan, Kansas & $39^{\circ} 14^{\prime}$ & $96^{\circ} 35^{\prime}$ & 68 & 28 \\
\hline \multirow[t]{2}{*}{46} & Morgantown, & & & & \\
\hline & W.-Va & $39^{\circ} 46^{\prime}$ & $79^{\circ} 56^{\prime}$ & 460 & 33 \\
\hline 42 & Medaryville, Ind. & $40^{\circ} 07^{\prime}$ & $86^{\circ} 52^{\prime}$ & 123 & 23 \\
\hline 38 & Nebraska City, Ne. & $40^{\circ} 46^{\prime}$ & $95^{\circ} 48^{\prime}$ & 134 & 25 \\
\hline 24 & Frémont, Ohio & $41^{\circ} 21^{\prime}$ & $83^{\circ} 07^{\prime}$ & 130 & 12 \\
\hline 45 & Des Moines, Iowa & $41^{\circ} 43^{\prime}$ & $93^{\circ} 35^{\prime}$ & 75 & 11 \\
\hline 39 & Kellogg Ft. Mich. & $42^{\circ} 22^{\prime}$ & $85^{\circ} 22^{\prime}$ & 141 & 13 \\
\hline 35 & Rutland, Vt. & $43^{\circ} 37^{\prime}$ & $72^{\circ} 58^{\prime}$ & 92 & 9 \\
\hline 49 & Oka, Qué. & $45^{\circ} 29^{\prime}$ & $74^{\circ} 02^{\prime}$ & 196 & 一 \\
\hline 20 & Pembroke, Ont. & $45^{\circ} 48^{\prime}$ & $76^{\circ} 53^{\prime}$ & 350 & 一 \\
\hline 12 & Pte Platon, Qué. & $46^{\circ} 40^{\prime}$ & $71^{\circ} 51^{\prime}$ & 269 & - \\
\hline 17 & Westbank, B.C. & $49^{\circ} 50^{\prime}$ & $119^{\circ} 35^{\prime}$ & 161 & - \\
\hline
\end{tabular}

dernière diminue progressivement à mesure qu'augmente la latitude du lieu jusqu'à un seuil acceptable qui se situe sensiblement au $43^{\circ} 37^{\prime}$ (Vermont). Les provenances originant d'une latitude inférieure au $43^{\circ}$ de latitude nord risquent de ne jamais $s^{\prime}$ adapter et celles-ci furent éliminées du programme de plantation. Notons que les deux provenances de I'Ontario utilisées dans la vieille plantation, sont d'une latitude sensiblement voisine de celle du Vermont et de ce fait peuvent être considérées comme marginales. Ce qui explique d'une part la disparition d'un grand nombre de semis entre les années 18821884 (I'auteur ensemença plus de 10,000 noix) et l'existence d'autre part, d'une réserve de variabilité due à une certaine proportion d'allèles hétérozygotes, qui confère à une espèce forestière donnée une flexibilité (plasticité) devant l'écart entre les conditions écologiques du lieu d'origine et celles du lieu de la plantation (cliché 1). Notons que les descendants des individus sélectionnés (phénotypes sains et à fort rendement en volume ligneux) n'ont pas souffert jusqu'à date du climat et poussent normalement.

Cette confrontation de la population résiduelle de noyer noir avec son nouveau milieu met en relief un gradient de rusticité formé sous l'influence de la sélection naturelle. C'est un nouvel état d'équilibre avec ce milieu aboutissant à une nouvelle adaptation. Ce fut un hasard heureux que les noix utilisées furent d'une telle latitude: des provenances quelque peu plus méridionales n'auraient pu résister, la limite du seuil de résistance ayant été dépassée. Cette hypothèse fut ainsi vérifiée au moyen de l'essai de 17 provenances mises en cause durant les hivers 1967 et 1968 . Ces saisons furent normales à la moyenne c'est-à-dire sans écart subit de température, sans dégel hivernal nocif aux noix ensemencées, et sans froids tardifs qui auraient pu nuire aux jeunes plantules. 


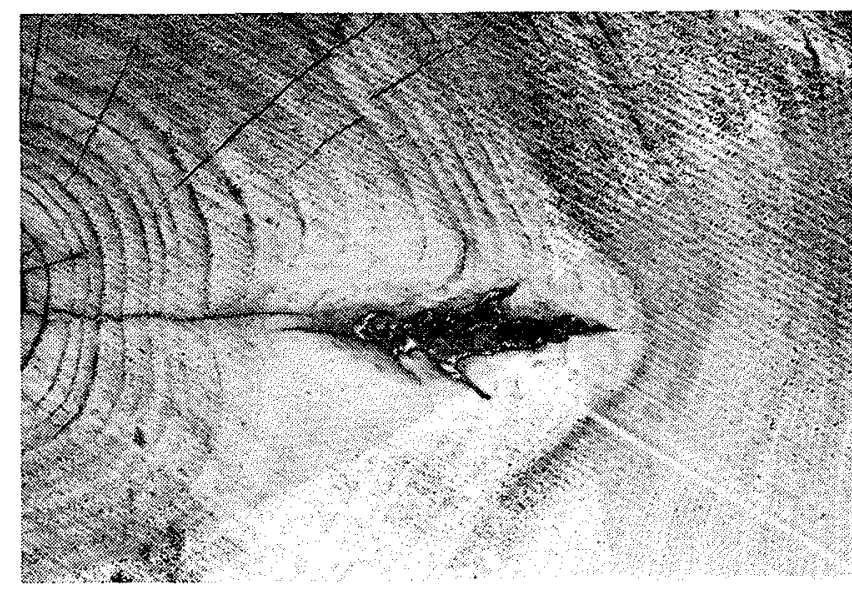

Cliché 1. Coupe transversole d'une rondelle de noyer noir (hauteur 65 pieds, diamètre 15 po.) âgé de 90 ons et montrant un début de gélivure recouvert par les tissus de cicatrisation.

\section{c) Conclusions}

Si un programme d'introduction d'une espèce exotique comprend plusieurs problèmes de base, (le choix de l'espèce, les connaissances de l'écologie de l'espèce dans son aire géographique et celles du nouveau milieu d'utilisation, les conditions climatiques locales, etc.), l'expérimentation sur le terrain permet, dans une très large mesure, d'évaluer la capacité finale de production de cette dernière en son nouveau milieu, et surtout sa plasticité qui joue un rôle fondamental dans de tels essais. Le même principe vaut pour une essence non introduite mais dont les réactions à la culture sont peu connues et la variabilité spécifique ignorée.

Cet exemple du noyer noir nous permet d'espérer que les meilleurs individus de certaines provenances peuvent produire un volume ligneux satisfaisant. Ceux-ci reproduits suivant une certaine échelle pourraient satisfaire à plusieurs entreprises locales, surtout si un programme de plantation se situe au sud du $46^{\circ} 40^{\prime}$ sans négliger les conditions édaphiques ainsi que celles du site du lieu de culture.

Une espèce aussi recherchée que le Juglans nigra, soit le Juglans cinerea peut être incluse avec profit dans un programme d'amélioration. En effet cette espèce autochtone en nos régions est plus plastique que la première, étant moins marginale. Elle présente de ce fait moins de difficultés à être cultivée d'un point de vue adaptation climatologique surtout. Une étude génécologique actuellement en cours et groupant des provenances réparties dans tout le nord-est de l'aire naturelle de l'espèce permettra de sélectionner les meilleures sources de noix à utiliser à l'avenir à l'intérieur d'une région définie. Les meilleures régions d'utilisation des semences étant connues, des essais par familles pourront être établis. Amélioreurs et sylviculteurs peuvent par la suite mettre leurs connaissances en commun à profit pour la phase définitive: la plantation. La deuxième consultation mondiale de la FAO et de IUFRO tenue à Washington, D.C., en août 1969, a traité à plusieurs reprises de l'importance de connaître la variabilité des espèces forestières à utiliser sous différentes latitudes de diffé- rents continents. Les recommandations techniques finales, lors des consultations de Stockholm (1963) et de Washington, D.C. (1969) également, citent entre autre le besoin en études génécologiques des principales espèces forestières utilisées en différents pays.

L'amélioration des arbres a un rôle essentiel à jouer dans nombre de pays où l'afforestation prend une importance toujours croissante. L'amélioreur doit planifier ses travaux pour aujourd'hui, demain et même prévoir pour les 10 décennies à venir.

\section{4- Une étude sur l'Acer saccharum}

\section{a) Avant-propos}

L'industrie printanière que procure l'érable à sucre est connue de tous et permet à un grand nombre de personnes d'augmenter leurs revenus annuels à une période où les travaux extérieurs touchant beaucoup de fermiers sont encore arrêtés par la fin de l'hiver.

Depuis l'époque où les Amérindiens du nord-est de l'Amérique entaillaient à leur façon les tiges de cette espèce, les techniques de récolte, de production et de conservation ont largement évolué et subissent encore de nos jours maints changements qu'une recherche active en ce domaine met au point avec les années que nous traversons.

\section{b) La notion de l'espèce}

L'espèce Acer saccharum, comme toute espèce forestière est constituée d'individus et de groupes d'individus qui évoluent selon leur potentiel héréditaire et le milieu. Plusieurs critères comparatifs peuvent être étudiés soit sur une grande échelle ou suivant une échelle plus restreinte. Ce genre d'études, auxquelles peuvent se joindre les recherches biosystématiques, commencent à aboutir à la connaissance de la variation à l'intérieur d'une espèce ligneuse donnée, variation au sens large d'une part et à la différenciation locale d'autre part. Nous sommes donc passés aujourd'hui de la conception qui considérait l'espèce comme une entité immuable d'individus uniformes (qui prévalait au temps de Linné) à la conception néo-darwinienne de l'espèce qui permet de découvrir la part héréditaire de la variation à l'intérieur des espèces.

\section{c) L'érable à sucre: espèce variable}

Le but de la présente étude consista à chercher s'il existait une variation importante locale dans la teneur en sucre de la sève ou "eau d'érable" recueillie au printemps et qui justifierait une sélection individuelle à l'intérieur d'un peuplement donné ou d'une région définie. Les individus caractérisés par une forte teneur en sucre pourraient alors être reproduits végétativement et constituer des clones améliorés. Est-ce là cependant un caractère stable à héritabilité élevée? Certains facteurs du milieu variant d'un printemps à l'autre, entraînent-ils une variation importante chez les mêmes individus étudiés suivant ce rapport de la teneur en sucre dans la sève? 
Certains auteurs étudièrent brièvement la variation de la teneur en sucre de l'érable (9); établirent des techniques de tests précoces des génotypes de l'érable à sucre et firent une première approche sélective (7). En 1962-63, des relevés plus méthodiques furent effectués afin de connaître si une sélection était justifiable pour des génotypes susceptibles de donner une sève à forte teneur en sucre. Pendant ces périodes propices à un premier inventaire de la variation possible du pourcentage de sucre dans la sève des tiges, l'auteur utilisa plusieurs érablières des états du Vermont, du New Hampshire, du Maine et de New York. II sélectionna ainsi plusieurs individus caractérisés par une teneur en sucre dépassant de façon significative la moyenne d'une population donnée (3).

\section{d) Matériel et Méthode}

Durant le printemps de 1965, et jusqu'en 1969, trois érablières furent choisies dans trois comtés différents, soit une à Ste-Catherine, Comté de Portneuf (no 1), une seconde près de Loretteville. Comté de Chauveau (no 2) et la troisième, à l'lle d'Orléans, Comté de Montmorency (no 3 ).

Dès que le mécanisme physiologique démarrait au printemps de 1965, un premier relevé fut effectué dans les peuplements en question, choississant ici et là des arbres d'aspect et de dimensions sensiblement voisins.

Cette estimation de la teneur en sucre des tiges était effectuée au moyen d'un réfractomètre Bausch and Lomb, qui permet d'obtenir des lectures suivant une précision de $0.1 \%$.

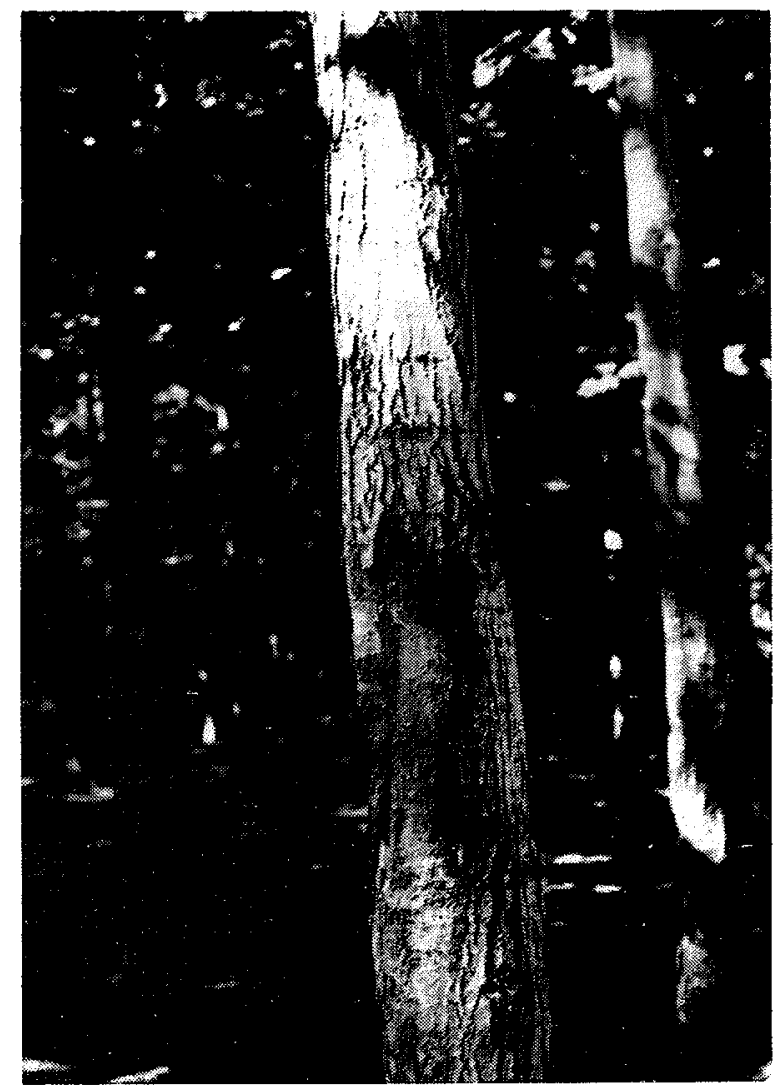

Cliché 2. - Individu No. 27 de l'érablière 2, dont la moyenne générale de la teneur en sucre est de $7.2 \%$. Houteur 51 pieds; d.h.p., 6.5 pouces.
En se basant sur la valeur moyenne généralement obtenue qui se fixe près de $3 \%-3.5 \%$ de sucre dans la sève, un arbre qui donnait une teneur en sucre supérieure à $30 \%$ de plus que ses 5 voisins immédiats, et de $1 \%$ supérieure au plus sucré des arbres témoins, satisfaisait nos critères sélectifs et se trouvait retenu pour la présente étude afin de constater si oui ou non cette valeur se maintenait de jour en jour durant la même saison, puis d'une saison à l'autre. Cette première approche faite au hasard calcula ainsi le critère recherché sur plus de 650 tiges. Après cette première analyse rapide, effectuée dans l'érablière no 2, 50 tiges par peuplement furent jugées suffisantes et conservées pour la durée de la présente étude. Chaque arbre était muni d'une étiquette d'aluminium numérotée pour faciliter le travail et identifier ces derniers. D'année en année, les lectures furent prises sur les mêmes individus, et la totalité des données quotidiennes enregistrées en moins d'une heure, à l'intérieur d'un même peuplement et ce, afin de diminuer le plus possible les variations qui se produisent rapidement à cette époque avec le temps du jour. La visite des érablières s'effectuait régulièrement de l'ouest à l'est, commençant par le peuplement situé à l'ouest de Québec pour se terminer par celui de l'lle d'Orléans.

\section{e) Quelques résultats}

Parmi les 150 érables à sucre visités pendant ces 5 années, 10 ont conservé de façon constante des valeurs en sucre de la sève répondant aux critères de sélection fixés. Ils se situent ainsi: érablière no

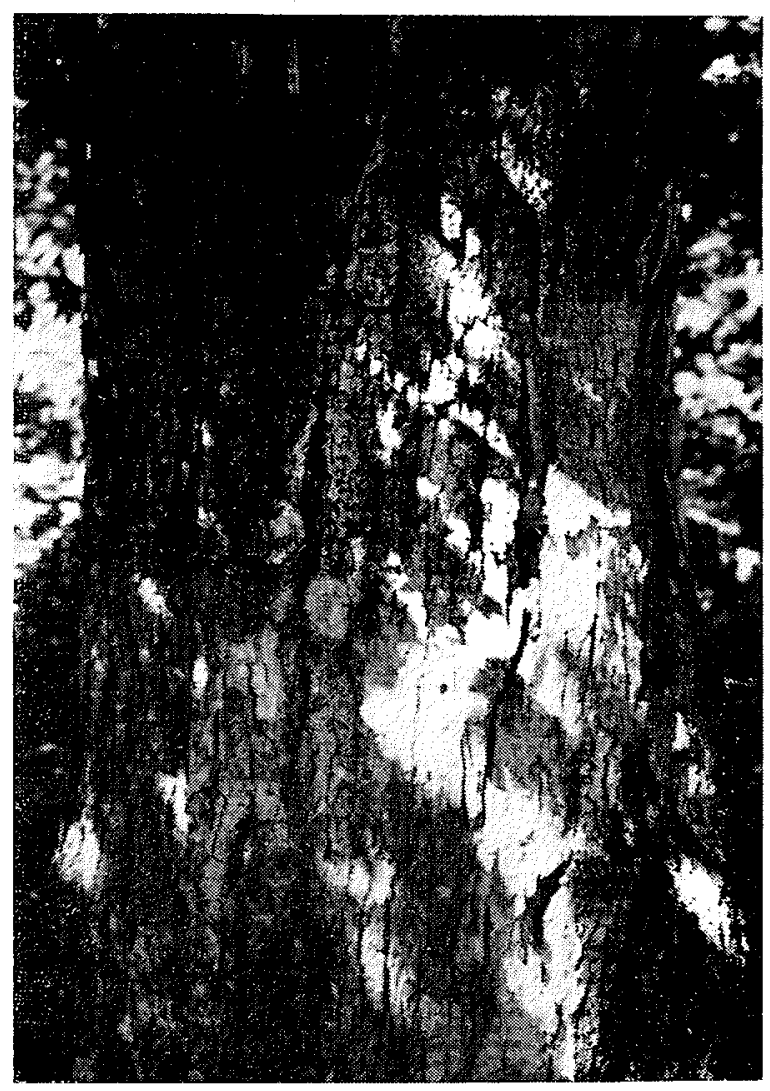

Cliché 3. - Individu No 50 de l'érablière 1 donnant une teneuur en sucre de $5 \%$. Houteur 66 pieds; d.h.p., 26 pouces. 
1: 1 ; érablière no 2 : 9 ; érablière no $3: 0$. Toutes les valeurs extraites des tiges des érablières 1 et 3 sont demeurées de façon constante et homogène près de la moyenne générale de $3-3.5 \%$, à l'exception d'un individu du peuplement 1 dont la moyenne se chiffre à $5 \%$.

Dans la troisième érablière la variation de la teneur en sucre fut très hérétogène et peut se résumer ainsi: 29 individus produisent une sève dont la moyenne quinquennale demeure fixe à $3-3.5 \%$. A l'opposé, 9 tiges se caractérisent par une teneur en sucre élevée. Ces valeurs oscillent entre $4.6 \%$ et $9.7 \%$ et sont significativement différentes de la valeur de la moyenne générale $(3.5 \%)$ suivant un seuil de probabilité de $99 \%$. Quant aux valeurs résiduelles, elles dépassent $3.5 \%$ mais demeurent inférieures à $4.6 \%$ (12 arbres). Celles-ci ne fournissent pas une valeur de la teneur en sucre qui soit différente de la moyenne statistiquement parlant.

\section{f) Conclusions}

Les 3 peuplements d'érable à sucre retenus pour la présente recherche se situent sur des terrains plats ou légèrement en pente et jouissant d'une exposition analogue. Les classes d'âge sont également sensiblement rapprochées bien qu'il ne semble pas y avoir de relation entre l'âge et la teneur en sucre (6). Si on fait abstraction de l'unique individu qui peut être sélectionné dans l'érablière no 1 , et qui s'est développé un peu à l'écart du peuplement, les résultats des peuplements 1 et 3 sont nettement différents de ceux obtenus dans le peuplement 2. Ce peuplement contient $18 \%$ d'individus dont la teneur en sucre varie de façon intéressante non seulement entre ces neuf tiges, mais davantage entre le reste des individus du peuplement. On constate ici l'importance de cette variation lorsqu'on sait que la quantité de sève nécessaire à la production d'un volume donné de sirop dépend de la teneur en sucre de la sève lorsque celle-ci quitte l'arbre. Ainsi une sève caractérisée par une teneur en sucre de $7.5 \%$ par exemple (rare) requiert trois fois moins de soluté pour produire un gallon de sirop qu'une sève dont la teneur se chiffre à $2.5 \%$.

Les individus caractérisés par une forte teneur en sucre, d'année en année, permettent de croire qu'il s'agit d'un caractère héritable. En effet, ces arbres poussent à proximité de voisins à faible teneur et doivent normalement jouir des mêmes conditions de sol. L'étude des cimes et d'espacement ne conduit pas non plus à des différences marquées. Avec la méthode de travail employée, la sélection s'est effectuée principalement d'un

Ouvrages consultés

$$
\text { L-Avant propos et Juglans }
$$

(1) BOUVAREL, P. 1960 - Les vieux pins laricio greffés de la Forêt de Fontainebleau. Silvae Genetica 9: $41-44$.

(2) DORMAN, K. W. 1969 - Positive impacts of silviculture on gene resources. Second World Consultation of Forest Tree Breeding, Washington, D.C. FO-FTB-69-10/3. point de vue génotypique plutôt que phénotypique, diminuant le plus possible l'influence du milieu. Toute sélection individuelle devient alors pratique lorsque basée sur une telle variation où les différences observées peuvent être héritables. L'érable à sucre est une espèce répandue dans tout le nordest de l'Amérique du Nord et pour une telle espèce, il serait opportun de connaitre les limites de cette variation de la teneur en sucre par régions définies. Si de telles populations se délimitaient par des valeurs différentes de régions voisines, la sélection se concentrerait davantage en de tels endroits. Les valeurs présentées ici semblent être les plus fortes obtenues à date: serait-il possible que ce caractère présente une variation plus importante lorsque l'espèce tend vers sa limite septentrionale?

La variation observée dans l'une des trois érablières indique bien un caractère variant également dans plusieurs cas d'arbre en arbre. Cette variation se prolonge d'année en année et même si un individu à forte teneur en sucre fournit lui-même une légère variation d'un printemps à l'autre, cette dernière ne varie pas suffisamment pour le déclasser et entraîner son rejet comme arbre à sélectionner suivant les critères utilisés. Le gain génétique se chiffre chez les individus les plus intéressants dans l'ordre de $150 \%$. Si on sait que les gènes simples à caractère qualitatif contrôlent les caractères spécifiques et sont liés de façon permanente à la structure de l'espèce, on ne connaît pas encore tout des gènes simples à caractère quantitatif contrôlant les caractères fluctuants d'un groupe d'individus.

Afin de déterminer si cette première estimation de la variation observée est définitivement génétique, des essais comparatifs de provenances et de clones sont indispensables. (Les méthodes de propagation végétative de l'érable à sucre n'ont pas encore donné à date de résultats valables et pratiques).

En effet, tout programme d'amélioration peut contenir deux phases. La première, connaître pour une espèce donnée la variation d'un caractère défini et les possibilités de sélection d'un meilleur matériel au sein d'une telle population variable. La seconde, propager le matériel ainsi retenu par les techniques d'amélioration tout en tenant compte des informations sylvicoles. Ces applications ainsi mises de l'avant aideront à développer, pour une espèce donnée, une sylviculture intensive avec laquelle le potentiel génétique peut être réalisé en termes de profit. D'autres études viendront sans doute apporter plus de lumière sur ce caractère plastique qu'est la teneur en sucre de la sève de l'Acer saccharum.

(3) FORESTRY CHRONICLE 1935 - Extracts pertaining to his black walnut plantations, from the diary of Sir Henri Joly de Lotbinière, XI, no 2 p. 81-91.

(4) GELLATLY, J. 1967-8 - Communication personnelle sur le noyer noir - Westbank, B.C.

(5) JOLY DE LOTBINIERE, Henri 1874-84 - Journal personnel. La Seigneurie de Lotbinière, Leclercville, Qué.

(6) LARSEN, S. 1956 - Genetics in Silvicuiture. Oliver and Boyd London, 225 p. V: 56-72. 
(7) MARSH, E. D. 1969 - Selecting adapted races of introduced species. Second World Consultation of Forest Tree Breeding, Washington, D.C., FO-FTB69-10/4

(8) PARROT L. 1968 - Les Provenances et leur intérêt dans le reboisement: Application au noyer noir. Texte de conférence, la Corporation des Ing. Forestiers, Prov. de Québec, $8 \mathrm{p}$.

(9) PARROT, L. 1969 - Le climat, facteur sélectif, et l'adaptation génétique de Juglans nigra, L., espèce exotique au Québec, Canada. Silvae Genetica (à l'impression).

(10) VON ALTHEN, F. W. 1965 - Hardwood planting problems and possibilities in Eastern Canada. Dept. of Forestry publication no 1043, 40p.

(11) VON ALTHEN F. W. 1969 - Plantations d'essences forestières feuillues dans le sud de l'Ontario. Ministère des Pêches et des Forêts du Canada, bull. no $1242 \mathrm{~F}$.

(12) WRIGHT, J.W. 1963 - Aspects génétiques de l'Amélioration des arbres forestiers. F.A.O, 16, 424 p.

\section{Il-Acer saccharum}

(1) Agricultural Research Service, U.S.D.A., 1968 - Pro- ceedings of the Seventh Conference on Maple Froducts, Philadelphia, $\mathrm{Pa} .48 \mathrm{p}$.

(2) FAFARD, A. 1968 - Communication personnelle

(3) GABRIEL, W. J. 1964 - Selection of sugar maples for high sugar content in sap - Development of Procedures. Proc, of the Eleventh Northeastern Forest Tree Improvement Conference. New Brunswick N.J. 1963

(4) GABRIEL, W. J. and HOWE, G. E. 1968 - Practical problems of a sugar maple selection program. Proceedings 15th Northeastern Forest Tree Improvement Conference, Morgantown, West Virginia.

(5) KRIEBEL, H. 1957 - Patterns of genetic variation in sugar maple. Ohio Agr. Experiment Station. Res. Bull. $791.56 \mathrm{p}$.

(6) KRIEBEL, H. 1960 - Selection and testing for sugar yield in Acer saccharum, L. Fifth World Forestry Congress Proceedings, Seattle, Wash.

(7) KRIEBEL, H. 1963 - Some Techniques for Early Diagnosis of genotype in Acer saccharum, L. Der Zuchter, 6 Sonderheft, p. 68-70.

(8) TAYLOR, F. H. 1956 - Variation in sugar content of Maple sap. Vermont Agric. Expt. Sta. Bull. 587.

\section{Some Historical Notes}

\section{on Forest Tree Breeding}

\section{in Canada}

\section{By J. L. FARRAR}

ODC $165.3 \div 902:(72)$

"From rather uncertain beginnings early in this century, tree breeding has expanded until it now represents a major part of forest research from coast to coast."

\section{Abstract}

Information is presented on the beginnings of tree breeding in Canada, especially the events which took place in the federal government departments prior to World War II. Emphasis is on personalities and organization rather than scientific results.

\section{Résumé}

Quelques notes historiques sur I'amélioration des arbres forestiers, au Canada

A titre de renseignement, ce travail fait l'historique cles travaux d'amélioration des arbres du Canada. L'histoire remonte au début des travaux et s'étend jusqu'à la deuxième guerre mondiale. L'auteur revoit le déroulement des événements, à l'intérieur des différents services du gouvernement central, regardant de plus près les personnalités et l'organisation constituée plutôt que les résultats scientifiques.

In this paper, tree breeding is given a broad interpretation. It includes any forestry activity which recognized that individuals within a species vary genetically. Thus the testing of seed lots from different geographical origins and the collection of seed from superior-looking trees are included. Introduction of exotic trees and vegetative propagation are considered because of their close relationship to most tree improvement programs.

It was logical that some of the earliest Canadian work related to tree breeding took place on the Prairies. Here the new settlers longed for trees to give protection from wind and sun. Trials were conducted with native trees of various origins, and exotics were introduced from other parts of the
Faculty of Forestry, University of Toronto.

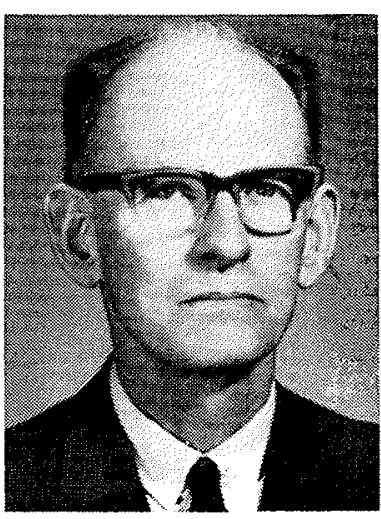

world. Beginning in the first decade of the twentieth century, the Canada Department of Agriculture began raising tree seedlings for prairie planting. Mr. Norman Ross was largely responsible for the success of this program $(39,40,41)$. Work in breeding trees for prairie planting has been continued under Mr. J. Walker and the present superintendent of the Tree Nursery Division at Indian Head, Saskatchewan, Dr. W. H. Cram (7,9). Tribute must also be paid to Mr. F. L. Skinner - a private nurseryman who did much to provide prairie farmers with suitable strains of trees, shrubs, fruits, and flowering plants $(38,42)$. He collected in many foreign countries and created new hybrids. His work with Larix, Populus, and Tilia was especially noteworthy.

Also in the early 1900's there were a number of advocates of tree planting in eastern Canada, most prominent of whom was Sir Henri Joly de Lotbinière $(1,32)$. On his seigniory near Pointe Platon, Quebec, he planted walnut and white pine using plants from a number of sources. In 1940 some of the white pine were found to be free of blister rust and were used as parents in breeding rustresistant trees (31).

in the $1920^{\prime} \mathrm{s}$, Messrs. Ellwood Wilson, R. W. Lyons and G. G. Cosens, working for the Laurentide Power and Paper Company, set out extensive plantations near Grand Mere, Quebec. They recognized the importance of seed source and the characteristics of the parent trees from which seeds were collected (26).

In general, Canadian foresters were slow to rec-" ognize the importance of tree breeding even though 